\title{
Design of Active Inductor Based Tunable Low Noise Amplifier (LNA)
}

\author{
V.Subhashini ${ }^{1}$, Dr.M.Maheswari ${ }^{2}$, A.Abinaya ${ }^{3}$ \\ Assistant Professor Department of ECE, K.Ramakrishnan College of Engineering, Trichy ${ }^{1}$ \\ Professor Department of ECE, K.Ramakrishnan College of Engineering, Trichy ${ }^{2}$ \\ Assistant Professor Department of ECE, K.Ramakrishnan College of Engineering, Trichy ${ }^{3}$
}

\begin{abstract}
In this paper, we proposed the design of active inductor based tunable Low Noise Amplifier. The circuit consists of an efficient active inductor. The performance of LNA is validated by its S-Parameter analysis and Noise Figure (NF). In order to decrease the silicon area and to enhance the Quality factor $(\mathrm{Q})$ of the LNA, the efficient active inductor is proposed and its performance is determined through post layout simulation using ADS tool. The designed active inductor based tunable LNA is operated in lower band frequency ranges from 3-5 GHz. The designed LNA operates at supply voltage of $1.2 \mathrm{~V}$. The proposed active inductor was designed by using a 180nm CMOS technology, which gives improved quality factor as high as 30 with a $7.9 \mathrm{nH}$ at $4 \mathrm{GHz}$. The proposed LNA shows up to $47 \%$ improvement in gain and up to $43 \%$ improvement in Noise Figure. The proposed LNA shows better performance for Sparameter measurements.
\end{abstract}

Keywords: Ultra Wide Band (UWB), Noise Figure (NF), Low Noise Amplifier (LNA), Active Inductor (AI), Complementary Metal Oxide Semiconductor (CMOS)

\section{INTRODUCTION}

Radio Frequency (RF) transmitters and recipients nowadays utilized as a part of various applications such as Global Positioning System (GPS), Wireless Fidelity (Wi-Fi), Bluetooth, RF Identification (RFID) frameworks etc.., The rapid growth of RF systems results in demand of low power and compact transceivers for wireless communication applications. UWB innovation has been intended to bring accommodation and versatility of rapid remote correspondence to homes and workplaces. Always UWB radio transmissions can legally operated in the frequency ranges from $3.1 \mathrm{GHz}$ up to $10.6 \mathrm{GHz}$, a short range pulses used for tracking various objects.

LNA is consider as the initial block of the receiver in order to strengthen the received signal with less noise. Using a LNA, the amount of noise from other upcoming stages of the receiver is decreased by improving the gain of LNA. To prevent the reflections of the signal between antenna and LNA, the input impedance value should be matched with the antenna impedance. To attain this, matching networks plays a important role at the input and output side of LNA. LNA design is mainly used because of minimum power, high gain and low NF. Different techniques have been recently introduced to achieve low power consumption with large gain and low NF. Inductive LNA is widely used for UWB application.

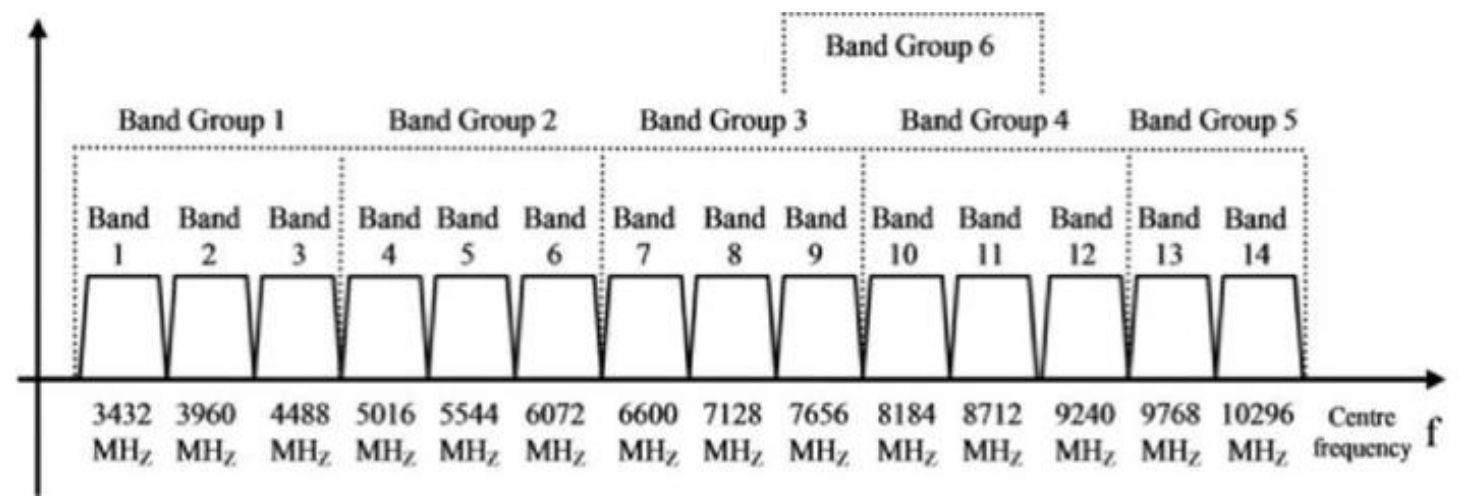

Fig 1.Spectrum allocations for UWB standard 


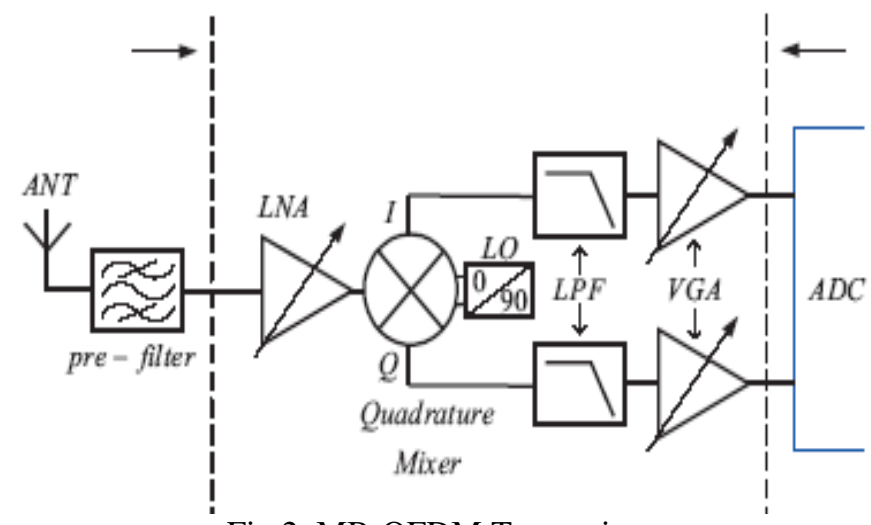

Fig 2. MB-OFDM Transceiver

As shown Fig 2, a typical MB-OFDM front-end consists of a LNA, LPF, Mixer and VGA. This UWB wireless receiver employs direct-conversion architecture and located behind the antenna and pre-filter. In the receiver front end, the design of LNA plays very important role since it determines overall system performance.

\section{LITERATURE SURVEY}

Research outcomes from both the academic community and industry have been reported in several peer reviewed publications on seen LNA for UWB receiver. Some existing literatures are listed below, that were referenced included in the project and captured their essence individually, each offering its own unique features.

\section{A. LITERATURE SURVEY ON TUNABLE LNA}

Mohammad Arif Sobhan Bhuiyan ,et al., (2015) proposed an active inductor based CMOS LNA has been designed using $2.4 \mathrm{GHz}$ ISM band RF receivers. It provides a good gain, but the bandwidth is restricted only at $2.4 \mathrm{GHz}$, not covering the entire spectrum of UWB.

V.Vaithianathan, et al., (2013) proposed differential LNA is proposed with 90nm CMOS technology and implemented which gives total power gain of more than $13 \mathrm{~dB}$ throughout the UWB spectrum. Hence power consumption of designed LNA is very huge due to increased number of stages.

Loay Khalaf, et al.,(2013) implemented active inductors which is implemented using tuned low noise amplifiers to reduce area. This method is widely used in personal wireless links such as Zigbee and Bluetooth.

Vibhor Kumar Bhardwaj, et al.,(2012) implemented LNA using active inductor with tunability under UWB range. The proposed design works only with single dc supply.

Maheswari, et al., (2015) proposed LNA using BJT amplifier for the frequency range of 5 to $6 \mathrm{GHz}$ and used RLC feedback and stability network to increase the stability of the amplifier. The author has not used active inductor in LNA.

Maheswari, et al., (2017) proposed a LNA using different filters for WBAN network. The authors have not used active inductor for performance improvement of LNA.

\section{B. LITERATURE SURVEY ON ACTIVE INDUCTOR}

Mrs. Amruta A.Ranade (2015) implemented active inductor circuit which can substantially improve its inductance value. The proposed design is implemented with $180 \mathrm{~nm}$ CMOS technology, which gives a quality factor of 28 with a $6.7 \mathrm{nH}$ at $4 \mathrm{GHz}$.

J.Manjula, et al., (2012) implemented a low power tunable active inductor suitable for designing Low noise amplifier. The designed active inductor was simulated in 0.18 CMOS technology by using HSPICE simulation tool. Simulation results gives an inductive bandwidth ranges from $1.5 \mathrm{MHz}$ to $7.94 \mathrm{GHz}$.

F.Gong, et al., (2010) proposed a high Quality factor Differential type active inductor with wide tuning range. The measured self resonant frequency is tunable ranges from $0.5 \mathrm{GHz}-10.2 \mathrm{GHz}$.Quality Factor of the proposed system is as high as 3000.But the inductor is intrinsically non-linear.

Through the Literature survey, it is evident that a better tunable LNA is required for UWB applications. The research interest in the appearance of tunable LNA making an receiver works better in terms of Low Noise Figure in a large frequency range grows quickly. 
Vol. 8, Issue 5, May 2019

\section{PROPOSED ACTIVE INDUCTOR}

An active inductor is basically an implementation of an inductor by using MOSFETs. A CMOS active inductor uses both NMOS and PMOS transistors. But spiral inductors achieves factors of low quality and large die area. In order to overcome this, active inductors are widely used as they have unique features like reduce area and also used in realizing circuits like filters and tuned amplifiers. To achieve large inductance value, passive inductor requires less silicon area and high quality factor $(\mathrm{Q})$ which is impractical. Hence active inductor is widely used.

The performance of an active inductor is analyzed using its figure-of-merits. CMOS active inductors have variety of applications in radio frequency integrated circuit (RFIC) design, which includes filters, phase shifters, oscillators, LNA and power dividers.

When $C g s$ is considered, $C_{1}=C g s_{2}, G_{o 1} \approx g_{o 1}+g o_{2}, G m 1=g_{m 2}, C_{2}=C g s_{1}, G o_{2}=1 / g_{m l}$, and $G m_{2}=g m_{2}$. The parameters of the equivalent RLC network of proposed active inductor are analyzed.

A current reuse active inductor with high quality factor is introduced by $\mathrm{Wu}$ which avoids the use of expensive passive inductors. From that, we are designed an active inductor based on Wu's current reuse technique. The overall schematic diagram of Wu's active inductor is shown in Fig 3.

It is given that the parameters of the RLC equivalent circuit of the designed active inductor are given by,
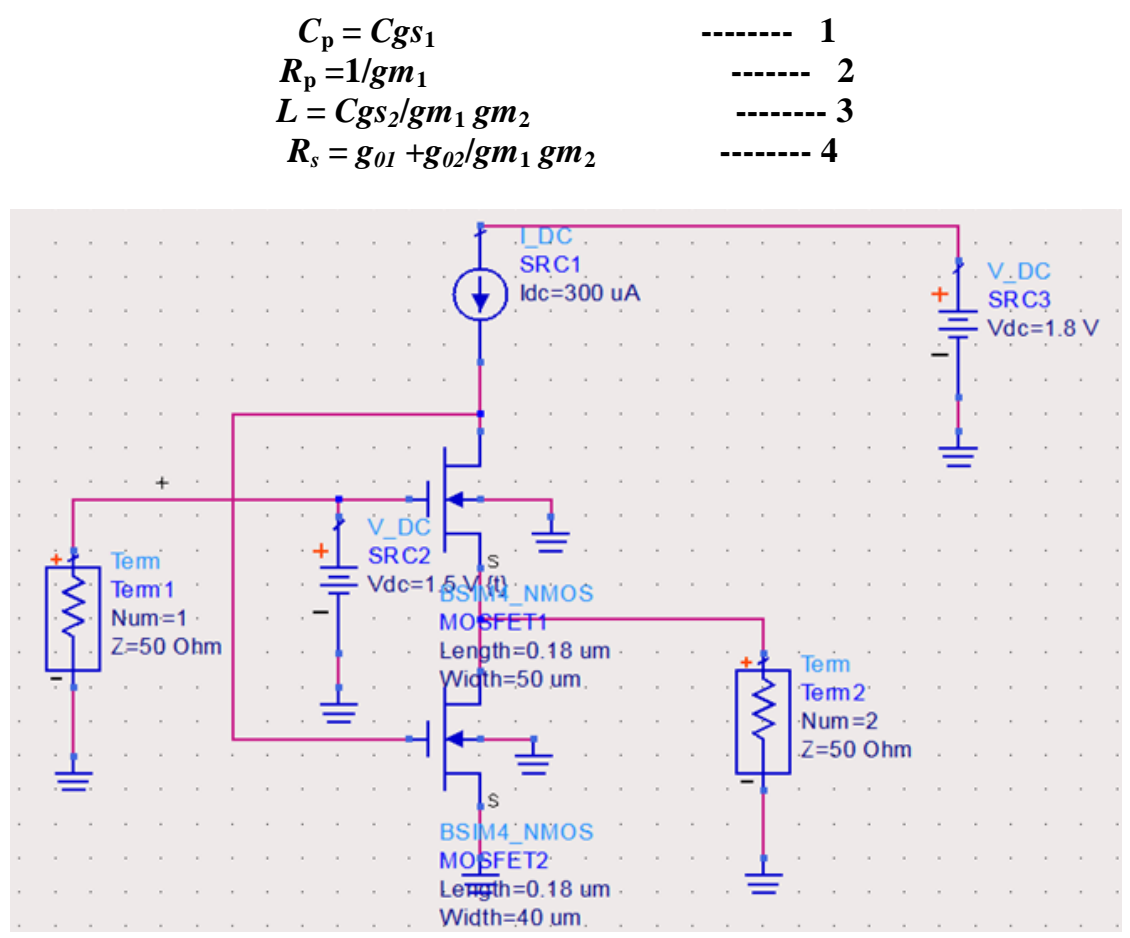

Fig 3. Schematic of proposed active inductor

From the above equation, the value of inductance L, the parasitic series resistance Rs, and parasitic parallel resistance $\mathrm{Rp}$ are depends on $\mathrm{g}_{\mathrm{m} 1}$ and $\mathrm{g}_{\mathrm{m} 2}$, which are calculated by the channel current value of transistor $\mathrm{M}_{1}$ and $\mathrm{M}_{2}$. Hence the value of quality factor of the proposed active inductors can be calculated by neglecting the value of Rs and only focusing on $\mathrm{Rp}$ as $\mathrm{Rp}$ is very small.

$$
\mathbf{Q}=\mathbf{R}_{\mathrm{p}} / \mathbf{w L}
$$

\section{PROPOSED LNA DESIGN}

In a receiver, LNA is basically act as a first signal processing module after the antenna. By considering all the favorable features of the overall systems some challenges still exist for the realization of LNA. It is capable of amplify all the received signals without any distortion. The sensitivity of LNA affects the overall sensitivity of receiver end. Another important requirement of the LNA is to maintain high gain and to reduce the noise of subsequent blocks.

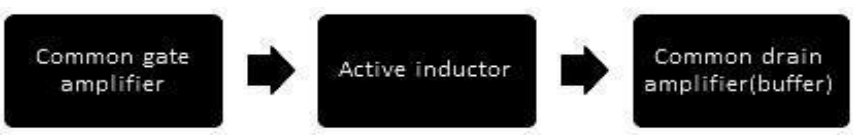

Fig.4. Proposed LNA structure 
Vol. 8, Issue 5, May 2019

The proposed LNA is structured in such a way that above three modules are interconnected, as shown in Fig.4. The signal will initially enter in to common gate topology and then into the active inductor and finally into a common drain topology which act as a buffer. All three modules are connected and the overall schematic diagram of the designed tunable LNA is illustrated in Fig.5. From the schematic, the active inductor module is formed by transistors $\mathbf{M}_{6}$ to $\mathbf{M}_{3}$ and the common gate amplifier design is formed by transistor $\mathbf{M}_{8}$. Hence, the transistor $\mathbf{M}_{7}, \mathrm{M}_{1}$ and $\mathbf{M}_{0}$ act as constant current source. Finally, transistor $\mathrm{M}_{9}$ and $\mathrm{M}_{10}$ formed the output buffer. It is well known that amplifiers add little noise and distortion to the desired received signal. Therefore, analysis of noise is very much important in any amplifier circuit. Noise figure is the important figure of merit which is used to measure the amount of noise which is added to a signal and is transmitted through the network. For any circuit achieving minimum noise figure is very essential. But it is already known that maximum gain and minimum noise cannot be obtained at the same time. Here transistor $\mathbf{M}_{11}$ is commonly configured with Common Gate configuration to maintain the trans-conductance of the amplifying transistor. The existence of that transistor does not have any changes in performance of LNA. By grounding the bias voltage, there is no change in centre frequency of transistor $\mathrm{M}_{9}$. But. LNA gain improvement is observed.

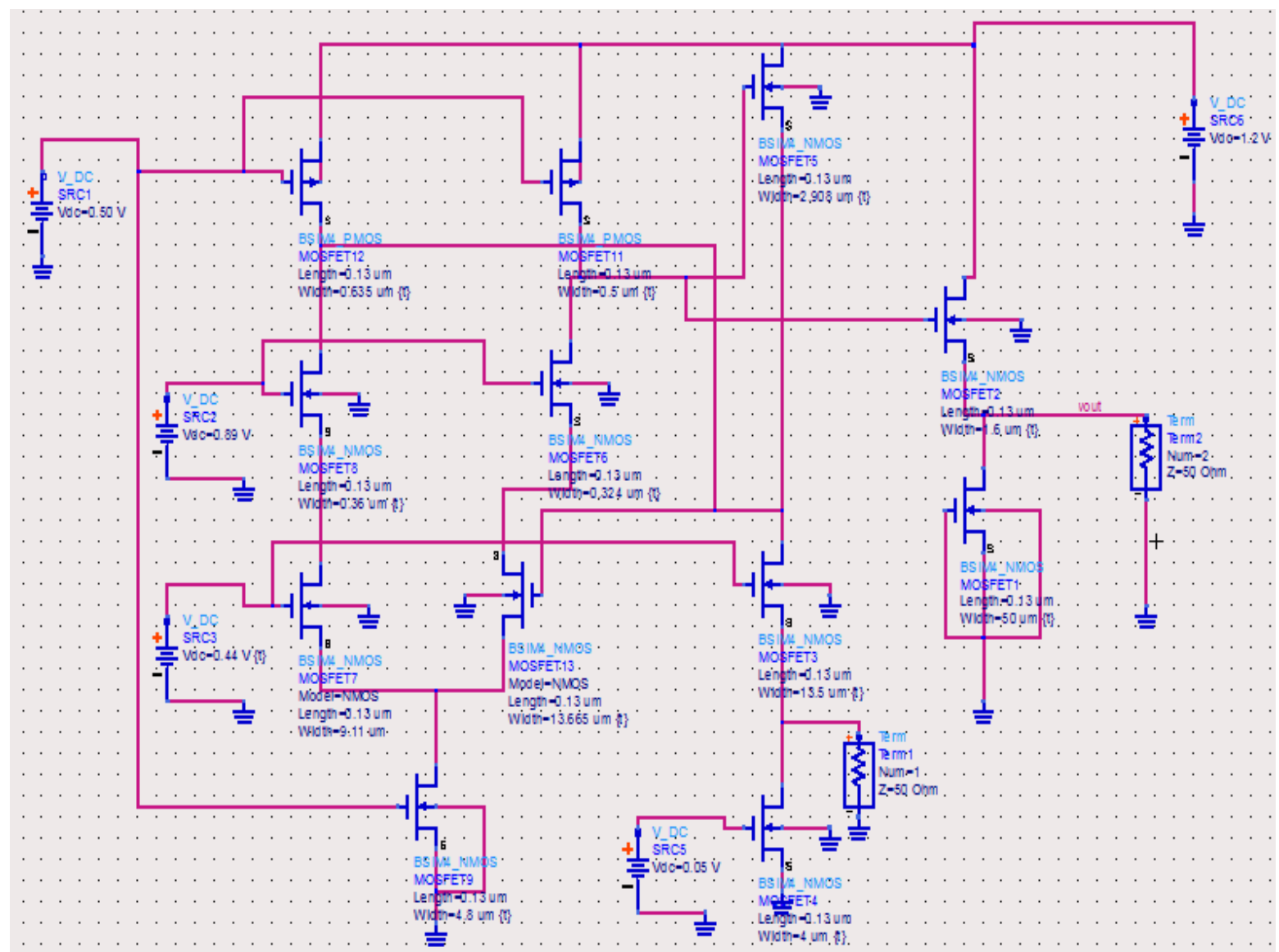

Fig.5. Schematic of active inductor based tunable LNA.

All the transistors in this design are operated under saturation region except for transistor $\mathrm{M}_{9}$. By altering the width of the transistor $\mathrm{M}_{2}, \mathrm{M}_{3}, \mathrm{M}_{4}, \mathrm{M}_{8}$ and $\mathrm{M}_{10}$, the figure of merit of LNA can be tuned. It is noted that the minimum NF is more as the gain increases. The main focus is to achieve high gain which intern produces minimum NF by tuning transistor width.

\section{SIMULATION RESULTS AND ITS DISCUSSION}

For analysis, the complete proposed design has been implemented by using ADS simulation tool. All the parameters included in this design is corresponds to $180-\mathrm{nm}$ CMOS technology. The power supply used is $1.2 \mathrm{~V}$ and the operating temperature of the circuit is under $300 \mathrm{~K}$ for measuring noise figure of LNA.

Input Reflection Coefficient $\left(\mathbf{S}_{\mathbf{1 1}}\right)$ : It is impossible to get both noise and power matching simultaneously in an LNA design. Its typical value should be less than $-10 \mathrm{~dB}$ while low noise figure is maintained. Fig 6.,shows the input matching for proposed design is $<-10 \mathrm{~dB}$. 


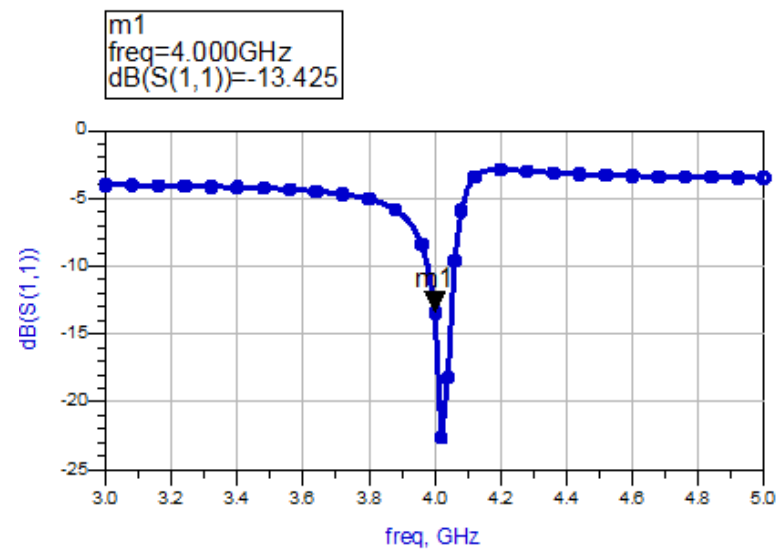

Fig.6. Input matching $\left(\mathrm{S}_{11}\right)$ of the LNA schematic

Reverse Isolation $\left(\mathbf{S}_{12}\right)$ : The reverse isolation $\left(S_{12}\right)$ is very crucial parameter to give better stability. The $S_{12}$ in plotted in the Fig.7.The proposed design has reverse isolation value of $<-34 \mathrm{~dB}$ for the above proposed LNA.

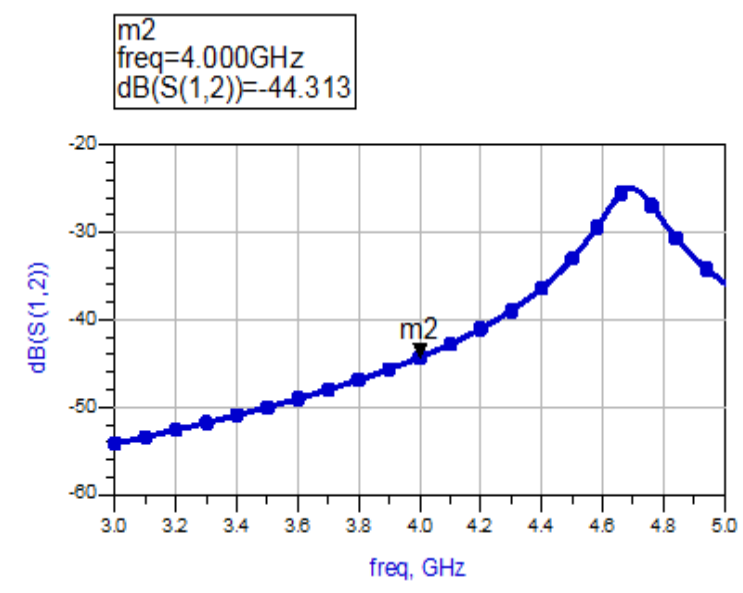

Fig.7. Reverse Isolation $\left(\mathrm{S}_{12}\right)$ of the LNA schematic.

Power Gain $\left(\mathbf{S}_{\mathbf{2 1}}\right)$ : The LNA should have large power gain in order to reduce the noise value in the subsequent stages at the receiver front end. From the Fig.8, the proposed circuit has achieved maximum gain greater than 10dB.

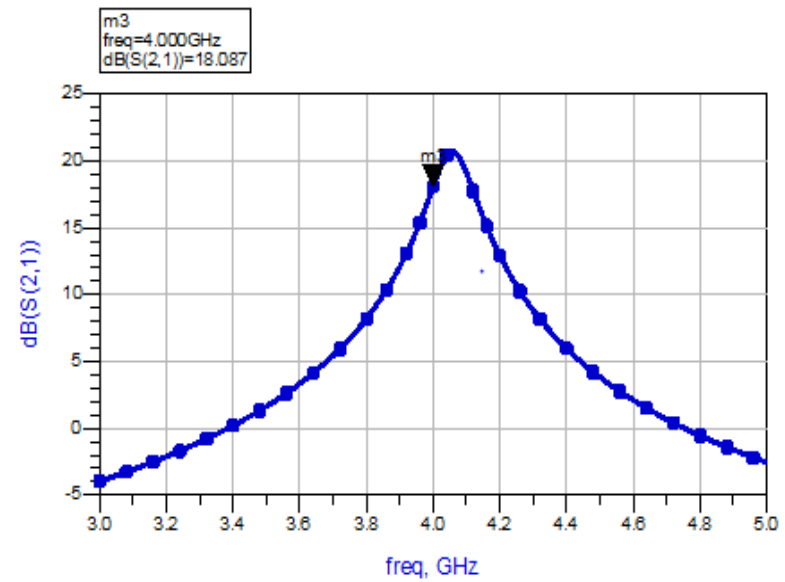

Fig.8. Power gain $\left(\mathrm{S}_{21}\right)$ of the LNA schematic.

Output Matching $\left(\mathbf{S}_{\mathbf{2} 2}\right)$ : It is very important to know that the output matching network does not alter the DC bias of the active circuit. From Fig.9, it is inferred that the output matching for proposed design is <-10dB. 


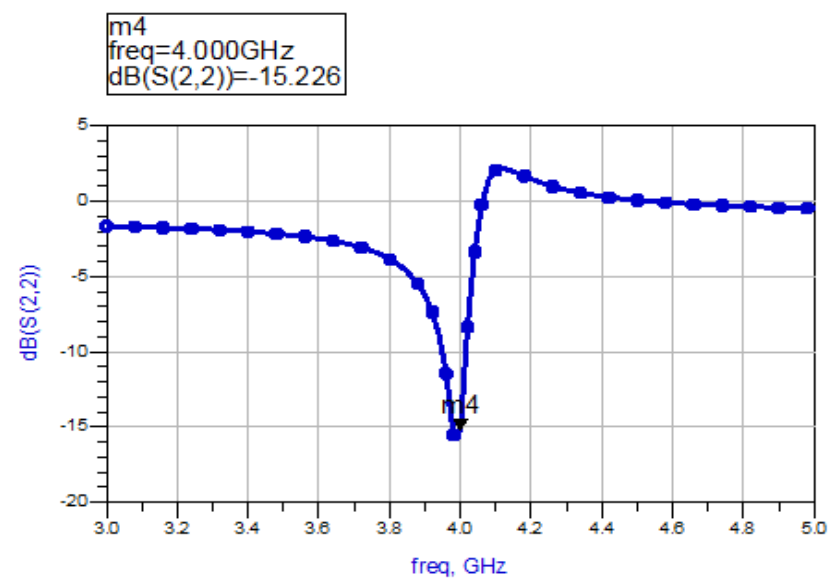

Fig.9. Output Matching $\left(\mathrm{S}_{22}\right)$ of the LNA schematic.

Noise Figure (NF min): The primary role of the LNA is to reduce the overall noise figure of RF front-end. Ideally it should be less than $3 \mathrm{~dB}$.Active inductors in general induce some self generated noise. As we have employed Active Inductor to reduce the chip area, the noise figure gets increased. From the Fig.10, the proposed design has a noise figure lesser than $4.8 \mathrm{~dB}$.

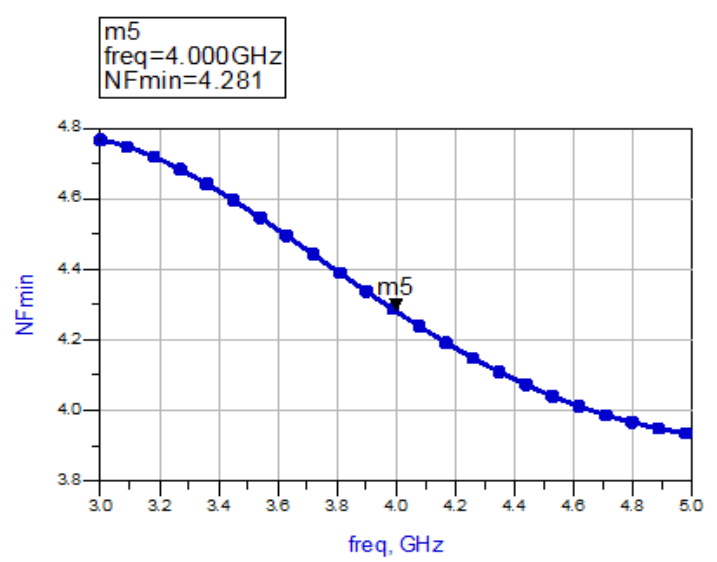

Fig.10. Noise Figure (NFmin) of the LNA schematic.

Quality Factor: The quality factor $(\mathrm{Q})$ is defined as the ratio of its inductive reactance to its resistance at a given frequency. The higher the $\mathrm{Q}$ factor of the inductor, then the inductor value reaches certain level. The proposed design employed here provides a improved quality factor of 30 throughout UWB frequency shown in Fig.11.

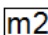

freq $=4.000 \mathrm{GHz}$ $\mathrm{Qd}=30.629$

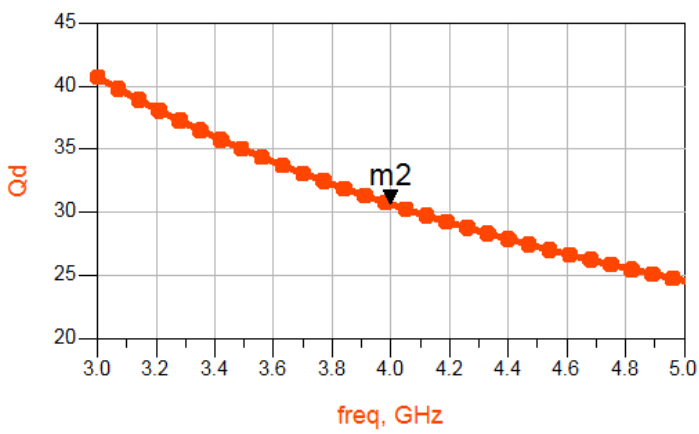

Fig.11. Quality factor vs Frequency

Inductance: The inductance of differential active inductor is positive throughout the range. The value of inductance in active inductor ranges from $4 \mathrm{n} \mathrm{H}-14 \mathrm{n} \mathrm{H}$ throughout the UWB is shown in Fig. 12. 
Vol. 8, Issue 5, May 2019

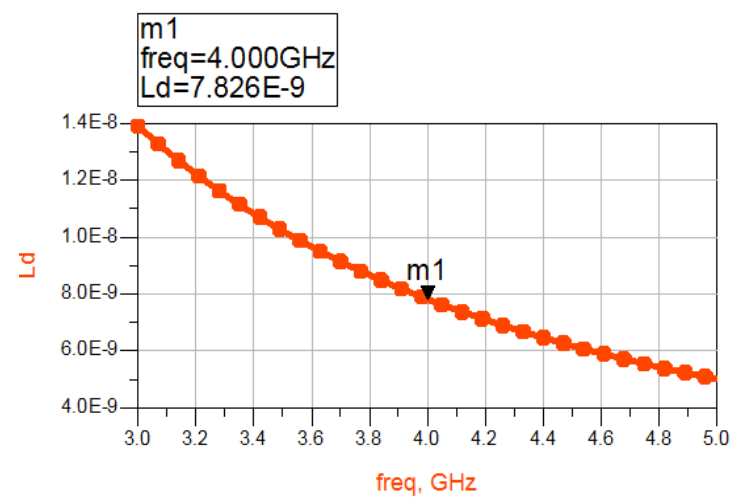

Fig.12.Inductance Vs Frequency

Table.1 Simulation Results Summary and Performance Analysis

\begin{tabular}{|l|c|c|c|c|}
\hline Parameters & [6] & {$[2]$} & {$[1]$} & Proposed LNA \\
\hline Technology & $180 \mathrm{~nm}$ & $180 \mathrm{~nm}$ & $180 \mathrm{~nm}$ & $180 \mathrm{~nm}$ \\
\hline Gain $\mathrm{S}_{21}(\mathrm{~dB})$ & 9.7 & 11 & 9.8 & 18.1 \\
\hline NFmin $(\mathrm{dB})$ & 7.5 & 5 & 4.5 & 4.28 \\
\hline Input Matching $\left(\mathrm{S}_{11}\right) \mathrm{dB}$ & $<-10$ & $<-12$ & $<-9$ & $<-14$ \\
\hline Reverse Gain $\mathrm{S}_{12}(\mathrm{~dB})$ & $<-30.5$ & NA & $<-20.8$ & $<-44.3$ \\
\hline Output Matching $\mathrm{S}_{22}(\mathrm{~dB})$ & $<-11.5$ & $<-14$ & $<-10$ & $<-15.2$ \\
\hline
\end{tabular}

VI.

CONCLUSION

From the proposed design, it is concluded that efficient active inductor based tunable LNA is designed using 180-nm CMOS technology. The proposed active inductor for LNA improves the quality factor. Active inductors in general induce some self generated noise. It should also have higher inductance value, wide frequency tuning range and higher quality factor(Q) which are essential for design of LNA. The proposed current reuse active inductor topology improves its equivalent inductance value. This designed active inductor also implemented by using 180-nm CMOS technology, which gives a maximum quality-factor of 30 with high inductance value of $7.9 \mathrm{nH}$ at $4 \mathrm{GHz}$. The proposed active inductor based tunable LNA results appreciable forward gain $\left(\mathrm{S}_{21}\right)$ of $18 \mathrm{~dB}$, high reverse isolation $\left(\mathrm{S}_{12}\right)$ of $-44.1 \mathrm{~dB}$, output matching $\left(\mathrm{S}_{22}\right)$ of $-15.4 \mathrm{~dB}$ and input matching $\left(\mathrm{S}_{11}\right)$ of -13 at $4 \mathrm{GHz}$. The LNA operates at supply voltage of $1.2 \mathrm{~V}$. However, the LNA shows a competitive NF of $4.21 \mathrm{~dB}$. Hence, the proposed is very much suitable for high performance, fully integrated, low cost and low power RF receiver designs.

\section{REFERENCES}

[1]. Bhardwaj V.K, Agarwal .A and Kumar .H , "CMOS LNA based on tunable active inductor for UWB applications",Interanational Conference on Power, Control and Embedded Systems, pp. 1-3,2012.

[2]. Gao.M, Wang.Y, Wang.Y and Guo.D, "Design of wideband CMOS LNA with active inductor and using noise-canceling technique", International Conference on Anti-counterfeiting, Security, and Identification in Communication, 2009 (ASID 2009), pp. $262-265$.

[3]. Krishnamurthy.S.V, El-Sankary K, El-Masry.E, "Noise-cancelling CMOS active inductor and its application in RF band-pass filter design",International Journal of Microwave Science and Technology, 2010, pp. 1-8.

[4]. M. Maheswari, R. Gayathri and S. Vimal, "Design and performance analysis of low noise amplifier with filters for WBAN based health monitoring system", Pakistan Journal of Biotechnology, Vol 14(1),pp.49-54,2017.

[5]. M. Maheshwari, Vimal.S, "Improved Design of RF BJT Low Noise Amplifier for 5 to 6 GHz Frequency Range",International Journal of Applied Engineering Research, International Journal of Applied Engineering Research ol 10,Issue 20,pp. 19969-19973,2015.

[6]. Malek .M.I and Saini .S "Improved two stage Ultra Wide Band CMOS low noise amplifier without band rejection using low noise active inductor", International Conference on Signal Processing and Communication Engineering Systems (SPACES-2015), pp. 157-161,2015.

[7]. Manjula .J and Malarvizhi .S, "Low power low noise tunable active inductor for narrow band LNA design", International Journal of Computer Applications, 47(17), pp. 39-43,2012.

[8]. Praveen .M, Venkatesan .V.Raja .J.Srinivasan , "Active Inductor Based Differential Low Noise Amplifier for Ultra Wide Band Applications", ICRTIT, Vol.44, No.19, pp. 401-406,2012.

[9]. Reja .M, Filanovsky .I, and Moez .K , "A CMOS 2.0-11.2 GHz UWB LNA using Active Inductor Circuit”, IEEE Int. Symp. On Circuits and Systems (ISCAS),pp. 281-284,2011.

[10]. Tang F,Yuan E., "Noise-cancelling CMOS active inductor and its application in RF band-pass filter design",Law Analog Integtator Circuit Signal Processing (2009) vol:58,pp.77-80,2009.

[11]. Weng .R .M, Liu .C.Y and Lin.P.C , "A low-power full-band low-noise amplifier for ultra-wideband receivers", IEEE Transaction on Microwave Theory and Techniques, 58(8), pp. 2077-2083,2009.

[12]. Yuan.F,Active Inductors and Transformers, Springer, 2008.

[13]. M Jayalakshmi, G Asha and K Keerthana, Control of Single Phase Z-Source Inverter Fed Induction Motor Using Simple Boost Controller, International Journal of Emerging Trends in Electrical and Electronics , vol.10,issue 10,pp.44-48, 2014. 